References

[1] L. Cesari, Sulle funzioni a variazione limitata, Annali Scuola. Norm. Sup. Pisa, Ser. 2, 5 (1936), pp. 299-313.

[2] - Sulle funzioni di due variabili a variazione limitata secondo Tonelli e sulla convergenza delle relative serie doppie di ITourier, Rend. di Matematica dell' Università di Roma 1 (1937), pp. 277-294.

[3] - Sulle funzioni di più variabili generalmente a variazione limitata e sullo convergenza della relative serie multiple di Fourier, Commentationes Pontificia Academia Sciontiarum 7 (1939), pp. 171-197.

[4] C. Goffman, Lower semicontinuity and area functions, T. The non-parametrio case, Rend. Circ. Mat. Palermo 2 (1953), pp. 203-235.

[5] J. Serrin, On the differentiabitity of functions of several variables, Archivo Rational Mechanics and Analysis 7 (1961), pp. 359-372

[6] A. M. Romano, Sul teorema di Jordan per le serie doppie di Fourier, Annali, Scuola Normale Sup. Pisa 12 (1943), pp. 85-97.

[7] L. Tonelli, Sulla quadratura delle superficie Atti, Accad. Naz. Lincei 3 (1926), pp. 357-363.

[8] - Sulla convergenza delle serie doppie d' Fourier, Annali di Matematica 4 (1927), pp. 29-72.

[9] - Sulle funzioni di due variabili generalmonte a variazione limitata, Annali Scuola Normale Sup. Pisa 5 (1936), pp. 315-320.

[10] - Sulle serie doppie di Fourier, Annali Scuola Normalo Sup. Pisa 6 (1937), pp. $315-326$

[11] G. Torrigiani, Sulle funzioni di piu variabili a variazione limitata, Riv. Mat. Univ. Parma 1 (1950), pp. 59-83.

[12] A. Zygmund, Trigonometric series, 2nd ed., Vol. 2, Chapter 17.

PURDUE UNIVERSTTY, WEST LAFAYETTE, INDIANA 47907 ACADHMIA SINIOA, NANKANG, TAIPEI, TATWAN 115

Received October 17, 1972

\section{On the continuity property of Gaussian random fields}

by

HISAO WATANABE (Fuknoka, Japan)

Abstract. The conditions for sample paths to be continuous are considered for Gaussian random fields. Especially, the necessary conditions are described.

§ 1. Introduction. Let $X=\left\{X(\bar{t}), \bar{t} \in \boldsymbol{R}^{d}\right\}$ be a zero mean, real, stationary, separable, mean continuous, Gaussian random field with a $d$ dimensional Euclidean parameter space. Then, the covariance function $\varrho(\bar{t})=E(X(\bar{t}+\bar{x}) X(\bar{s}))$ is expressed by $\int_{\boldsymbol{z}} \cos (\bar{t}, \bar{\lambda}) d F^{\prime}(\bar{\lambda})$, where $($,$) denotes$ the inner product, $\bar{t}, \bar{s} \in \boldsymbol{R}^{d}$ and $F^{(}(\cdot)^{\left({ }^{1}\right)}$ is a bounded positive measure.

The purpose of this paper is to describe the continuity condtitions of path functions (which are known for the 1-dimensional parameter case) for random fields. Most sufficient conditions for sample functions to be continuous are already described for random fields. Thus, we shall be concerned mainly with sufficient conditions for sample functions to be discontinuous.

In the case of the 1-dimensional parameter space, the conditions in terms of the spectral measure $F(\cdot)$ were given by Kahane [4] and Nisio [7]. The corresponding results for random fields are the following. Let $\quad s_{n}=F\left(\bar{\lambda} \in S_{2} n+1\right)-F\left(\bar{\lambda} \in S_{2} n\right), \quad$ where $\quad S_{2} n+1=\left\{\lambda ;|\bar{\lambda}| \leqslant 2^{n+1}\right\}$, $n=0,1,2, \ldots$

Trooxam 1. If $X(\bar{t})$ is continuous, then $\sum_{n=1}^{\infty} s_{n}^{\dagger}<\infty$.

Trmomism 2. If there exists a decreasing sequence $\left\{M_{n}\right\}$ such that $s_{n} \leqslant M_{n}$ and $\sum_{n=1}^{\infty}, M_{n}^{\frac{1}{\alpha}}<\infty$, then $X$ has continuous paths.

As is shown by Mareus [5] and. Marcus and Shepp [6], these conditions are neither too strong, nor necessary and sufficient. However, they give a simple criterion for some cases. In $\S 2$ and $\S 3$, we shall give the proof of the above theorems.

A result corresponding to theorem of Marcus and Shepp ([6], p. 380) is as follows.

(1) F is occasionally used as a measure or as a point function. 
THEOREM 3. Let $X$ be the above-mentioned Gaussian process and let $\{Y(r), r \in R\}$ be a stationary Gaussian process which is a restriction to a 1-dimensional parameter subspace of $X$. Let $\sigma^{2}(h)=E\left((Y(r+h)-Y(r))^{2}\right)$. Let $\psi$ be any nondecreasing local minorant of $\sigma$, that is, for some $\varepsilon>0$,

$$
\begin{gathered}
\sigma(h) \geqslant \psi(h) \geqslant 0, \quad 0 \leqslant h \leqslant \varepsilon, \\
\psi(h) \uparrow, \quad 0 \leqslant h \leqslant \varepsilon .
\end{gathered}
$$

If

$$
\int^{\infty} \psi\left(e^{-x^{2}}\right) d x=\infty
$$

then the paths of $X$ are not continuous.

Proof. The proof is easy. In $\S 4$, we shall deal with applications and comments.

A necessary and sufficient condition for Gaussian sample paths to be continuous is given by Sudakov [9], but it seems to be difficult to express his condition by any explicit formula involving the spectral function or the covariance function.

§ 2. Proof of Theorem 1. Let $\left\{T_{j}, j=1,2, \ldots\right\}$ be an increasing sequence of positive numbers such that $\sum_{j=1}^{\infty} \frac{1}{\sqrt{T_{j}}}<\infty$ and $T_{j} \geqslant 1$. Let $\chi(x)=\max (1-|x|, 0)$,

$\theta_{r}(\lambda)=\prod_{j=r}^{\infty} \chi\left(\frac{\lambda}{T_{j}}\right), \quad-\infty<\lambda<\infty$,

$\theta_{r}\left(\lambda_{1}, \ldots, \lambda_{d}\right)=\theta_{r}\left(\lambda_{1}\right) \theta_{r}\left(\lambda_{2}\right) \ldots \theta_{r}\left(\lambda_{d}\right), \quad-\infty<\lambda_{i}<\infty(i=1,2, \ldots, d)$,

$K_{r}(t)=\frac{1}{\sqrt{2 \pi}} \int_{-\infty}^{\infty} e^{i t \lambda} \chi\left(\frac{\lambda}{T_{r}}\right) d \lambda, \quad-\infty<t<\infty$,

$K_{r}\left(t_{1}, \ldots, t_{d}\right)=K_{r}\left(t_{1}\right) \ldots K_{r}\left(t_{d}\right), \quad-\infty<t_{i}<\infty(i=1,2, \ldots, d)$,

$l_{r}(t)=\frac{1}{\sqrt{2 \pi}} \int_{-\infty}^{\infty} e^{i t \lambda} \theta_{r}(\lambda) d \lambda, \quad-\infty<t<\infty$

$l_{r}\left(t_{1}, t_{2}, \ldots, t_{d}\right)=l_{r}\left(t_{1}\right) \ldots l_{r}\left(t_{d}\right), \quad-\infty<t_{i}<\infty(i=1,2, \ldots, d)$,

$l_{r}^{*}(t)=\frac{1}{\sqrt{2 \pi}} \int_{-T_{r-1}}^{T_{r-1}} e^{i t \lambda} \theta_{r}(\lambda) d x, \quad-\infty<t<\infty$,

$l_{r}^{*}\left(t_{1}, \ldots, t_{d}\right)=l_{r}^{*}\left(t_{1}\right) \ldots l_{r}^{*}\left(t_{d}\right), \quad-\infty<t_{i}<\infty(i=1,2, \ldots, d)$.
Hence we note that

$$
l_{r}\left(t_{1}, t_{2}, \ldots, t_{d}\right) \geqslant 0
$$

Also, we remark that

$$
l_{r}\left(t_{1}, \ldots, t_{d}\right)=\prod_{i=1}^{a}\left(l_{r+1} * K_{r}\right)\left(t_{i}\right) .
$$

As is well known, $X\left(t_{1} \ldots t_{d}\right)$ can be written in the form

$$
X\left(t_{1}, t_{2}, \ldots, t_{d}, \omega\right)=\int_{-\infty}^{\infty} \ldots \int_{-\infty}^{\infty} e^{i\left(t_{1} \lambda_{1}+t_{2} \lambda_{2}+\ldots+l_{d} \lambda\right)} d \Phi\left(\lambda_{1}, \lambda_{2}, \ldots, \lambda_{d}, \omega\right),
$$

where $\Phi(\cdot)$ is a Gaussian random measure.

Also, we put

$$
\begin{gathered}
Y_{r}\left(t_{1}, t_{2}, \ldots, t_{d}, \omega\right)=\left(\frac{1}{\sqrt{2 \pi}}\right)^{d} \int_{-\infty}^{\infty} \ldots \int_{-\infty}^{\infty} X\left(t_{1}-s_{1}, t_{2}-s_{2}, \ldots, t_{d}-s_{d}, \omega\right) \times \\
\quad \times l_{r}\left(s_{1}, s_{2}, \ldots, s_{d}\right) d s_{1} \ldots d s_{d}, \\
Y_{r}^{*}\left(t_{1}, t_{2}, \ldots, t_{d}, \omega\right)=\left(\frac{1}{\sqrt{2 \pi}}\right)^{d} \int_{-\infty}^{\infty} \ldots \int_{-\infty}^{\infty} X\left(t_{1}-s_{1}, t_{2}-s_{2}, \ldots, t_{d}-s_{d}, \omega\right) \times \\
\times l_{r}^{*}\left(s_{1}, s_{2}, \ldots, s_{d}\right) d s_{1} \ldots d s_{d} .
\end{gathered}
$$

We can rewrite $Y_{r}$ and $Y_{r}^{*}$ in the following way:

$$
\begin{aligned}
Y_{r}\left(t_{1}, t_{2}, \ldots, t_{d}, \omega\right)=\int_{-T_{r}}^{T_{r}} \ldots \int_{-T_{r}}^{T_{r}} e^{i\left(t_{1} \lambda_{1}+\ldots+t_{d} \lambda_{d}\right)} \theta_{r}\left(\lambda_{1}, \lambda_{2}, \ldots, \lambda_{d}\right) \times \\
\times d \Phi\left(\lambda_{1}, \lambda_{2}, \ldots, \lambda_{d}, \omega\right), \\
Y_{r}^{*}\left(t_{1}, t_{2}, \ldots, t_{d}, \omega\right)=\int_{-T_{r-1}}^{T_{r-1}} \cdots \int_{-r_{r-1}}^{T_{r-1}} e^{i\left(l_{1} \lambda_{1}+\ldots+t_{d} \lambda_{d}\right)} \theta_{r}\left(\lambda_{1}, \lambda_{2}, \ldots, \lambda_{d}\right) \times \\
\times d \Phi\left(\lambda_{1}, \lambda_{2}, \ldots, \lambda_{d}, \omega\right) .
\end{aligned}
$$

Since $X(t, \omega)$ is continuous with probability one, by Fernique [2] we obtain

$$
a=E\left(\sup _{\bar{t} \in[0,1]^{d}}|X(\bar{t})|\right)<\infty .
$$

We have the following lemma.

Lemina 2.1.

$$
E\left(\sup _{\bar{t} \in[0,1]^{d}}\left|Y_{r}^{*}(\bar{t})\right|\right) \leqslant E\left(\sup _{t \in[0,1]}\left|Y_{r}(\bar{t})\right|\right) \leqslant a .
$$


Proof. Let us put $Z_{r}\left(t_{1} \ldots t_{d}\right)=Y_{r}\left(t_{1} \ldots t_{d}\right)-Y_{r}^{*}\left(t_{1}, t_{2}, \ldots, t_{d}\right)$.

Then $Z_{r}$ has continuous paths and

$$
\begin{array}{r}
Z_{r}\left(t_{1}, t_{2}, \ldots, t_{d}\right)=\int_{\left(t_{1}, t_{2}, \ldots, t_{d}\right) \in \square T_{r}-\square T_{r-1}} e^{i\left(t_{1} \lambda_{1}+\ldots+t_{d} \lambda_{d}\right)} \theta_{r}\left(t_{1}, \ldots, t_{d}\right) \times \\
\times d \Phi\left(\lambda_{1}, \lambda_{2}, \ldots, \lambda_{d}\right),
\end{array}
$$

where $\square_{T}=\left\{\left(\lambda_{1}, \ldots, \lambda_{d}\right) ;\left|\lambda_{i}\right| \leqslant T(i=1,2, \ldots, d)\right\}$. Therefore, we can easily see that $Z_{r}+Y_{r}^{*}=Y_{r} ;$ moreover $Z_{r}$ and $Y_{r}^{*}$ are mutually independent as continuous function-valued random variables, and using Lemma 3.2.4.A in Delporte ([1], p. 143), we have

$$
E\left(\left\|Y_{r}^{*}\right\|\right) \leqslant E\left(\left\|Y_{r}\right\|\right)
$$

where $\|\cdot\|$ denotes the uniform norm of continuous functions. Since $\left(\frac{1}{\sqrt{2 \pi}}\right)^{d} \int_{-\infty}^{\infty} \ldots \int_{-\infty}^{\infty} l_{r}\left(s_{1}, s_{2}, \ldots, s_{d}\right) d s_{1} d s_{2} \ldots d s_{d}=1$, we have

$$
E\left(\left\|Y_{r}\right\|\right) \leqslant a .
$$

Define stationary Gaussian processes $V_{r}$ and $V_{r}^{*}$ by

$$
\begin{aligned}
& \nabla_{r}\left(t_{1}, t_{2}, \ldots, t_{d}\right)=\left(\frac{1}{\sqrt{2 \pi}}\right)^{d} \int_{\mathbb{\pi} i,\left|s_{i}\right| \geqslant \frac{1}{\sqrt{T_{r}}}} \ldots \int_{r+1}\left(t_{1}-s_{1}, t_{2}-s_{2}, \ldots, t_{d}-s_{d}, \omega\right) \times \\
& \times K_{r}\left(s_{1}, s_{2}, \ldots, s_{d}\right) d s_{1} d s_{2} \ldots d s_{d}, \\
& \nabla_{r}^{*}\left(t_{1}, t_{2}, \ldots, t_{d}\right)=\left(\frac{1}{\sqrt{2 \pi}}\right)^{d} \int_{\mathbb{\Psi} i,\left|s_{i}\right| \geqslant \frac{1}{\sqrt{T_{r}}}} \ldots Y_{r+1}^{*}\left(t_{1}-s_{1}, t_{2}-s_{2}, \ldots, t_{d}-s_{d}, \omega\right) \times \\
& \times K_{r}\left(s_{1}, \ldots, s_{d}\right) d s_{1} d s_{2} \ldots d s_{d} .
\end{aligned}
$$

The following estimates are crucial for the proof

$$
\begin{gathered}
K_{r}(t)=\frac{\sqrt{2}}{t^{2} T_{r} \sqrt{\pi}}\left(1-\cos T_{r} t\right) \geqslant 0, \\
\int_{\frac{1}{\sqrt{T_{r}}}}^{\infty} K_{r}(t) d t \leqslant \frac{2 \sqrt{2}}{\sqrt{\pi} \sqrt{T_{r}}}, \quad \int_{-\infty}^{\infty} K_{r}(t) d t=1, \\
\int_{\frac{1}{\sqrt{\bar{T}_{r}}}}^{\infty} l_{r}(t) d t \leqslant \frac{2 \sqrt{2}}{\sqrt{\pi} \sqrt{\overline{T_{r}}}} \quad \text { and } \quad \int_{-\infty}^{\infty} l_{r}(t) d t=1 .
\end{gathered}
$$

By making use of the above estimates, we have

and

$$
\begin{gathered}
\int_{\llbracket i,\left|s_{i}\right| \geqslant \frac{1}{\sqrt{\bar{r}_{r}}}} K_{r}\left(s_{1}, s_{2}, \ldots, s_{d}\right) d s_{1} d s_{2} \ldots d s_{d} \leqslant \frac{C}{\sqrt{T_{r}}} \\
\int_{\llbracket i,\left|s_{i}\right| \geqslant \frac{1}{\sqrt{T_{r}}}} l_{r}\left(s_{1}, s_{2}, \ldots, s_{d}\right) d s_{1} d s_{2} \ldots d s_{d} \leqslant \frac{C}{\sqrt{T_{r}}} .
\end{gathered}
$$

where $C$ is an absolute constant.

Therefore, we have

and

$$
\begin{aligned}
& E\left(\left\|V_{r}\right\|\right) \leqslant=\frac{C}{\sqrt{T_{r}}} \\
& E\left(\left\|V_{r}^{*}\right\|\right) \leqslant \frac{C}{\sqrt{T_{r}}}
\end{aligned}
$$

where $C$ is an absolute constant

LEMara 2.2 Let $\left\{T_{r}\right\}$ be an increasing sequence of positive numbers such that $\sum_{r=1}^{\infty} \frac{1}{\sqrt{T_{r}}}<\infty$ and $T_{r} \geqslant 1$. Then

$$
\sum_{j=1}^{\infty}\left(\int_{\left(\lambda_{1}, \lambda_{2}, \ldots, \lambda_{d}\right) \in \square_{T_{j+1}}} \ldots . \int_{\square_{T_{j}}} \prod_{k=j+1}^{\infty} \prod_{i=1}^{d}\left(1-\frac{\left|\lambda_{i}\right|}{T_{k c}}\right)^{2} d F\left(\lambda_{1}, \ldots, \lambda_{d}\right)\right)^{+}<\infty .
$$

Proof. We define successively the random variables $S_{j}, S_{j}^{\prime}$ and $H_{j}$ $j=1,2, \ldots$, as follows:

$$
S_{1}(\omega) \equiv 0
$$

$H_{1}(\omega) \equiv Y_{1}\left(S_{1}(\omega), \omega\right)$,

$$
\begin{aligned}
& \left\{\begin{array}{l}
\text { some } \bar{t}_{\in}\left\{\bar{t}_{1}=\left(t_{1}, \ldots t_{d}\right) ;\left|t_{i}\right| \leqslant \tau_{1},\right. \\
\left.\quad Y_{2}^{*}\left(t_{1}, \ldots, t_{d}, \omega\right)=\min _{\left(\left|s_{i}\right| \leqslant \tau_{1}(i=1,2, \ldots, a)\right)} Y_{2}^{*}\left(s_{\mathbf{1}}, s_{\mathbf{2}}, \ldots, s_{d}, \omega\right)\right\}
\end{array}\right. \\
& \text { if } H_{1}(\omega)<\min _{\left(\left|\delta_{i}\right| \leqslant \tau_{1}(i=1,2, \ldots, d)\right)} \bar{Y}_{2}^{*}\left(s_{1}^{\prime}, s_{2}, \ldots, s_{d}, \omega\right) \text {, } \\
& S_{1}(\omega) \equiv\left\{\operatorname { s o m e } \overline { t } \in \left\{\bar{t}^{\prime} ;\left|t_{i}\right| \leqslant \tau_{1}(i=1,2, \ldots, d),\right.\right. \\
& \left.Y_{2}^{*}\left(t_{1}, t_{2}, \ldots, t_{d}\right)=\max _{\left(\left|t_{i}\right|<\tau_{1}(i=1,2, \ldots, a)\right)} Y_{2}^{*}\left(s_{1}, s_{2}, \ldots, s_{d}, \omega\right)\right\} \\
& \text { if } H_{1}(\omega)>\max _{\left(\left|s_{i}\right|<\tau_{1}(i=1,2, \ldots, d)\right)} \Psi_{2}^{*}\left(s_{1}, s_{2}, \ldots, s_{d}, \omega\right) \text {, } \\
& \text { some } \bar{t} \in\left\{\bar{t} ;\left|t_{i}\right| \leqslant \tau_{1}(i=1,2, \ldots, d)\right. \text {, } \\
& \times\left(\mathbf{X}_{2}^{*}\left(t_{1}, \ldots, t_{a}, \omega\right)=\mathbb{B}_{\mathbf{1}}(\omega)\right\} \text {, otherwise, }
\end{aligned}
$$

where $\tau_{1}=1+\frac{1}{\sqrt{T_{1}}}$. 
Obviously, $\tilde{S}_{1}(\omega)$ is measurable with respect to the Borel field $\mathscr{B}_{3}$, generated by $\left\{d \Phi\left(\lambda_{1}, \lambda_{2}, \ldots, \lambda_{d}\right) ;\left|\lambda_{i}\right| \leqslant T_{1}(i=1,2, \ldots, d)\right\}$.

$$
S_{j+1}(\omega) \equiv\left\{\begin{array}{l}
\tilde{S}_{j}(\omega) \text { if } Y_{j+1}\left(\tilde{S}_{j}, \omega\right) \geqslant H_{j}(\omega), \\
\text { some } \bar{t} \epsilon\left\{\bar{t}=\left(t_{1}, t_{2}, \ldots, t_{d}\right) ;\left|t_{i}\right| \leqslant \tau_{j},\right. \\
\left.\quad Y_{j+1}\left(t_{1}, t_{2}, \ldots, t_{d}, \omega\right)=\max _{\left|s_{i}\right| \leqslant \tau_{j}} Y_{j+1}\left(s_{1}, s_{2}, \ldots, s_{d} ; \omega\right)\right\} \\
\quad \text { if } H_{j}(\omega)>\max _{\left\{\left|s_{i}\right| \leqslant \tau_{j}\right\}} Y_{j+1}\left(s_{1}, s_{2}, \ldots, s_{d}, \omega\right), \\
\text { some } \bar{t} \in\left\{\bar{t}=\left(t_{1}, t_{2}, \ldots, t_{d}\right) ;\left|t_{i}\right| \leqslant \tau_{j},\right. \\
\left.Y_{j+1}\left(t_{1}, t_{2}, \ldots, t_{d} ; \omega\right)=H_{j}(\omega)\right\}, \text { otherwise. }
\end{array}\right.
$$$$
H_{j+1}(\omega) \equiv \bar{j}_{j+1}\left(S_{j+1}(\omega), \omega\right) \text {. }
$$

$$
\tilde{S}_{j+1}(\omega) \equiv\left\{\begin{array}{c}
\text { some } \bar{t} \epsilon\left\{\bar{t}=\left(t_{1}, t_{2}, \ldots, t_{d}\right) ;\left|t_{i}\right| \leqslant \tau_{j+1},\right. \\
\left.Y_{j+2}^{*}\left(t_{1}, t_{2}, \ldots, t_{d}, \omega\right)=\min _{\left|s_{i}\right| \leqslant \tau_{j+1}} Y_{j+2}^{*}\left(s_{1}, \ldots, s_{d}, \omega\right)\right\} \\
\text { if } H_{j+1}(\omega)<\min _{\left|s_{i}\right| \leqslant \tau_{j+1}} Y_{j+2}^{*}\left(s_{1}, s_{2}, \ldots, s_{d}, \omega\right), \\
\text { some } \bar{t} \epsilon\left\{\bar{t}=\left(t_{1}, t_{2}, \ldots, t_{d}\right) ;\left|t_{i}\right| \leqslant \tau_{j+1},\right. \\
\left.Y_{j+1}^{*}\left(t_{1}, t_{2}, \ldots, t_{d}, \omega\right)=\max _{\left|s_{i}\right| \leqslant \tau_{j+1}} Y_{j+2}^{*}\left(s_{1}, s_{2}, \ldots, s_{d}, \omega\right)\right\} \\
\quad \text { if } H_{j+1}(\omega)>\max _{\left|s_{i}\right| \leqslant r_{j+1}} Y_{j+2}^{*}\left(s_{1}, s_{2}, \ldots, s_{d}, \omega\right), \\
\text { some } \bar{t} \in\left\{\bar{t}=\left(t_{1}, t_{2}, \ldots, t_{d}\right) ;\left|t_{i}\right| \leqslant \tau_{j+1},\right. \\
\left.Y_{j+2}^{*}\left(t_{1}, t_{2}, \ldots, t_{d}, \omega\right)=H_{j+1}(\omega)\right\}, \text { otherwise, }
\end{array}\right.
$$

where $\tau_{j}=1+\frac{1}{\sqrt{T_{1}}}+\ldots+\frac{1}{\sqrt{T_{j}}}$. By definition, $S_{j}$ and $\tilde{S}_{j}$ are measurable with respect to the Borel field $\mathscr{B}_{j}$, generated by $\left\{d \Phi\left(\lambda_{1}, \lambda_{2}, \ldots, \lambda_{d}\right)\right.$; $\left.\left|\lambda_{i}\right| \leqslant T_{j}\right\}$. Now we shall prove that

$$
P\left(\sup _{j=1,2, .}\left|H_{j}(\omega)\right|<\infty\right)=1 .
$$

Let us put

$$
\begin{aligned}
& Y_{r}\left(t_{1}, \ldots, t_{d}\right)=\left(\frac{1}{\sqrt{2 \pi}}\right)^{d} \int_{-\infty}^{\infty} \ldots \int_{-\infty}^{\infty} X\left(t_{1}-s_{1}, t_{2}-s_{2}, \ldots, t_{d}-s_{d}\right) \times \\
& \times l_{r}\left(s_{1}, s_{2}, \ldots, s_{d}\right) d s_{1} d s_{2} \ldots d s_{d} \\
&=\left(\frac{1}{\sqrt{2 \pi}}\right)^{d} \int_{\left|s_{i}\right| \leqslant \frac{1}{\sqrt{T_{r}}}} \ldots \int_{i=1, \ldots, d)} X\left(t_{1}-s_{1}, \ldots, t_{d}-s_{d}\right) \times \\
& \times l_{r}\left(s_{1}, \ldots, s_{d}\right) d s_{1} \ldots d s_{d}+
\end{aligned}
$$

$$
\begin{aligned}
&+\left(\frac{1}{\sqrt{2 \pi}}\right)^{d} \int_{\mathbb{I I} i,\left|s_{i}\right| \geqslant \frac{1}{\sqrt{T_{r}}}} \ldots \int_{r+1} X_{1}\left(t_{1}, \ldots, t_{d}-s_{d}\right) \times \\
& \times l_{r}\left(s_{1}, s_{2}, \ldots, s_{d}\right) d s_{1} d s_{2} \ldots d s_{d} .
\end{aligned}
$$

Here we can see that

$$
\begin{aligned}
& \sup _{\left|t_{i}\right| \leqslant \tau} \mid\left(\frac{1}{\sqrt{2 \pi}}\right)^{d} \int_{\left|s_{i}\right| \leqslant \frac{1}{\sqrt{x_{r}}}} \int_{(i=1, \ldots, d)} \ldots \int X\left(t_{1}-s_{1}, \ldots, t_{d}-s_{d}\right) \times \\
& \times l_{r}\left(s_{1}, s_{2}, \ldots, s_{d}\right) d s_{1}, \ldots, d s_{d}\left|\leqslant \sup _{\left|t_{i}\right| \leqslant 2 \tau}\right| X\left(t_{1}, \ldots, t_{d}\right) \mid
\end{aligned}
$$

with probability one; also, since $\int \ldots \int l_{r}\left(s_{1}, s_{2}, \ldots, s_{d}\right) d s_{1} d s_{2} \ldots d s_{d}$ $\leqslant$ const $/ \sqrt{T_{r}}$ we have

$$
\mathbb{G} i,\left|s_{i}\right| \leqslant \frac{1}{\sqrt{T_{r}}}
$$

$$
\begin{aligned}
\sum_{r=1}^{\infty} E\left(\sup _{\left|t_{i}\right| \leqslant \tau} \mid\left(\frac{1}{\sqrt{2 \pi}}\right)^{d} \int_{\mathbb{E I} i,\left|s_{i}\right| \geqslant}\right. & \frac{\int_{1}}{\sqrt{T_{r}}} X\left(t_{1}-s_{1}, \ldots, t_{d}-s_{d}\right) \times \\
& \left.\times l_{r}\left(s_{1}, s_{2}, \ldots, s_{d}\right) d s_{1} d s_{2} \ldots d s_{d} \mid\right)<\infty
\end{aligned}
$$

Therefore,

$$
\sup _{r} \sup _{\left|t_{i}\right| \leqslant \tau}\left|Y_{r}\left(t_{1}, \ldots, t_{d}\right)\right|<\infty \quad \text { with probability one, }
$$

which combined with the definition of $H_{j}$ yields (2.3). Now we observe the following relations:

$$
\begin{aligned}
H_{j+1}(\omega)-H_{j}(\omega)=\left\{\left(H_{j+1}(\omega)-\right.\right. & \left.\left.H_{j}(\omega)\right) \vee 0\right\}-\left\{\left(Y_{j}\left(S_{j}(\omega), \omega\right)-\right.\right. \\
& \left.\left.-\sup _{\left|\boldsymbol{s}_{i}\right| \leqslant \tau_{j}} Y_{j+1}\left(s_{1}, s_{2}, \ldots, s_{d}, \omega\right)\right) \vee 0\right\} .
\end{aligned}
$$

For $\left(t_{1}, t_{2}, \ldots, t_{d}\right)$ satisfying $\left|t_{i}\right| \leqslant \tau_{j-1}(i=1, \ldots, d)$, we have

$$
\begin{aligned}
Y_{j}\left(t_{1}, t_{2}, \ldots, t_{d}\right) \leqslant \sup _{\left|t_{i}\right| \leqslant \tau_{j}(i=1,2, \ldots, d)} Y_{j+1}\left(t_{1}, t_{2}, \ldots, t_{d}\right)+ & \\
& +\sup _{\left|t_{i}\right| \leqslant \tau_{j}-1} V_{j}\left(t_{1}, t_{2}, \ldots, t_{d}\right) .
\end{aligned}
$$

Therefore, for $\bar{t}$ such that $\left|t_{i}\right| \leqslant \tau_{j-1}(i=1,2, \ldots, d)$, we obtain

$$
\begin{gathered}
Y_{j+1}\left(t_{1}, t_{2}, \ldots, t_{d}\right)-\sup _{\left|s_{i}\right| \leqslant \tau_{j}} X_{j+1}\left(s_{1}, s_{2}, \ldots, s_{d}\right) \\
\quad \leqslant \sup _{\left|s_{i}\right| \leqslant \tau_{j-1}(i=1,2, \ldots, d)} V_{j}\left(s_{1}, s_{2}, \ldots, s_{d}\right), \\
\left(Y_{j}\left(S_{j}(\omega)\right)-\sup _{\left|s_{i}\right| \leqslant \tau_{j}} Y_{j+1}\left(s_{1}, s_{2}, \ldots, s_{d}\right)\right) \vee 0 \\
\leqslant \sup _{\left|s_{i}\right| \leqslant x_{j-1}(j=1,2, \ldots, d)}\left|V_{j}\left(s_{1}, s_{2}, \ldots, s_{d}\right)\right|
\end{gathered}
$$

with probability 1 . 
Again, by (2.1), we have

$$
\sum_{j=1}^{\infty} \mathbb{E}\left\{\left(Y_{j}\left(S_{j}\right)-\sup _{\left|s_{i}\right| \leqslant r_{j}(i=1,2, \ldots, d)} Y_{j+1}\left(s_{1}, s_{2}, \ldots, s_{d}\right)\right) \vee 0\right\}<\infty .
$$

By the use of (2.3) and the relation

$$
\begin{aligned}
& \sum_{j=1}^{n}\left(H_{j+1}-H_{j}\right) \vee 0 \\
& \quad=H_{n+1}-H_{1}+\sum_{j=1}^{n}\left(Y_{j}\left(S_{j}\right)-\sup _{\left|s_{i}\right| \leqslant \tau_{j}(i=1,2, \ldots, d)} Y_{j+1}\left(s_{1}, s_{2}, \ldots, s_{d}\right)\right) \vee 0,
\end{aligned}
$$

we have

$$
\sum_{j=1}^{\infty}\left(H_{j+1}-H_{j}\right) \vee 0<\infty, \quad \text { with probability } 1 .
$$

On the other hand,

$$
\begin{aligned}
\left(H_{j+1}-H_{j}\right) \vee 0 & =\left(Y_{j+1}\left(\tilde{S}_{j}\right)-H_{j}\right) \vee 0 \\
\geqslant & \left\{\left(Y_{j+1}\left(\tilde{S}_{j}\right)-Y_{j+1}^{*}\left(\tilde{S}_{j}\right)\right) \vee 0\right\}- \\
& \quad-\left\{\left(H_{j}-\sup _{\left|t_{i}\right| \leqslant \tau_{j}} Y_{j+1}^{*}\left(t_{1}, t_{2}, \ldots, t_{d}\right)\right) \vee 0\right\} .
\end{aligned}
$$

Also, observing that for $\left(t_{1}, t_{2}, \ldots, t_{d}\right)$ satisfying $\left|t_{i}\right| \leqslant \tau_{i-1}(i=1,2, \ldots, d)$

$$
\begin{aligned}
Y_{j}\left(t_{1}, t_{2}, \ldots, t_{d}\right) \leqslant \sup _{\left|t_{i}\right| \leqslant t_{j}(i=1, \ldots, d)} Y_{j+1}^{*}\left(t_{1}, t_{2}, \ldots, t_{d}\right)+ & \\
& +\sup _{\left|t_{i}\right| \leqslant t_{j-1}(i=1, \ldots, d)} V_{j}^{*}\left(t_{1}, t_{2}, \ldots, t_{d}\right),
\end{aligned}
$$

we have

$$
\begin{aligned}
&\left(Y_{j}\left(t_{1}, t_{2}, \ldots, t_{d}\right)-\sup _{\left|s_{i}\right| \leqslant r_{j}(i=1,2, \ldots, a)} X_{j+1}^{*}\left(s_{1}, s_{2}, \ldots, s_{d}\right)\right) \vee 0 \\
& \leqslant \sup _{\left|t_{i}\right|<\tau_{j-1}}\left|\nabla_{j}^{*}\left(t_{1}, t_{2}, \ldots, t_{d}\right)\right|
\end{aligned}
$$

and consequently

$$
\sum_{j=1}^{\infty} E\left(\left(Y_{j}\left(t_{1}, t_{2}, \ldots, t_{d}\right)-\sup _{\left|s_{i}\right| \leqslant \tau_{j}(i=1,2, \ldots, a)} Y_{j+1}^{*}\left(s_{1}, s_{2}, \ldots, s_{d}\right) \vee 0\right)\right)<\infty .
$$

Now, using (2.4) and the relation

$$
\begin{aligned}
\left(H_{j}(\omega)-\sup _{\left|t_{i}\right| \leqslant \tau_{j}} Y_{j+1}^{*}\left(t_{1}, t_{2}, \ldots, t_{d}\right)\right) \vee 0 \\
\quad=\left(Y_{j}\left(S_{j}(\omega), \omega\right)-\sup _{\left|t_{i}\right| \leqslant \tau_{j}} Y_{j+1}^{*}\left(t_{1}, t_{2}, \ldots, t_{d}\right)\right) \vee 0
\end{aligned}
$$

we get

$$
\sum_{j=1}^{\infty}\left(Y_{j+1}\left(\tilde{S}_{j}\right)-Y_{j+1}^{*}\left(\tilde{S}_{j}\right)\right) \vee 0<\infty \quad \text { with probability } 1 .
$$

Put

$$
\begin{aligned}
\gamma_{j} & =Y_{j+1}(\tilde{S})-Y_{j+1}^{*}\left(\tilde{S}_{j}\right) \\
& =\int_{\left(\lambda_{1}, \ldots, \lambda_{d}\right) \in \square_{T_{j+1}}-\square_{T_{j}}} \exp \left(i \sum_{i=1}^{a} \tilde{S}_{j}^{i} \lambda_{i}\right) \theta_{j+1}\left(\lambda_{1}, \ldots, \lambda_{d}\right) d \Phi\left(\lambda_{1}, \lambda_{2}, \ldots, \lambda_{d}\right)
\end{aligned}
$$

and

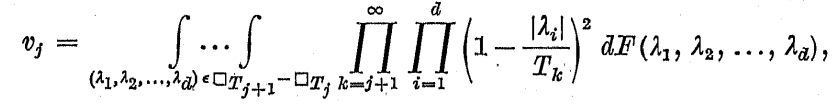

where $\tilde{S_{j}}=\left(\tilde{S_{j}^{1}}, \tilde{S}_{j}^{2}, \ldots, \tilde{S}_{j}^{d}\right)$. Then, in the same way as in Nisio [7], we obtain

$$
\sum_{j=1}^{\infty} \sqrt{v_{j}}<\infty
$$

which completes the proof of Lemma 2.2.

We shall now pass to the proof of Theorem 1 . Take $2^{-k}$ as $T_{k}$ and $\alpha=\prod_{k=0}^{\infty}\left(1-3 \cdot 2^{-k-2}\right)^{2 d}$.

Evidently, we get

$$
\begin{aligned}
& \alpha \int_{\left(\lambda_{1}, \ldots, \lambda_{d}\right) \in \square_{(3 / 2) 2^{j}}-\square_{2^{j}}} d F^{\prime}\left(\lambda_{1}, \lambda_{2}, \ldots, \lambda_{d}\right) \\
& \leqslant \int_{\left(\lambda_{1}, \ldots, \lambda_{d}\right) \in \square_{(3 / 2) 2_{j}^{j}}-\square_{2} j} \ldots \prod_{l_{i=j}=j+1+1}^{\infty} \prod_{i=1}^{d}\left(1-\frac{\left|\lambda_{i}\right|}{2^{k}}\right)^{2} d F\left(\lambda_{1}, \lambda_{2}, \ldots, \lambda_{d}\right) .
\end{aligned}
$$

Therefore

$$
\sum_{j=0}^{\infty}\left(\int_{\left(\lambda_{1}, \lambda_{2}, \ldots, \lambda_{d}\right) \in \square_{(3 / 2) 2_{2}^{j}} \square_{\square_{2} j}} d F\left(\lambda_{1}, \lambda_{2}, \ldots, \lambda_{d}\right)\right)^{1 / 2}<\infty .
$$

Also, let us talke $T_{k}=2 \cdot 2^{k-1}$ and $a=\prod_{k=0}^{\infty}\left(1-\frac{1}{8} 2^{-k+1}\right)^{2 d}$. 
Since

$$
\begin{aligned}
& 2 \alpha \underset{\left(\lambda_{1}, \lambda_{2}, \ldots, \lambda_{d}\right) \in \square_{2} j+1-\square_{(3 / 2) 2^{j}}}{ } d F\left(\lambda_{1}, \lambda_{2}, \ldots, \lambda_{d}\right) \\
& \leqslant \quad \int_{\left(\lambda_{1}, \lambda_{2}, \ldots, \lambda_{d}\right) \in \square_{2} j+1-\square_{2} j} \ldots \int_{k=j+1}^{\infty} \prod_{i=1}^{d}\left(1-\frac{\left|\lambda_{i}\right|}{3 \cdot 2^{k-1}}\right)^{2} d F\left(\lambda_{1}, \lambda_{2}, \ldots, \lambda_{d}\right),
\end{aligned}
$$

we have

$$
\sum_{j=1}^{\infty}\left(\int_{\left(\lambda_{1}, \ldots, \lambda_{d}\right) \in \square_{2} j+1-\square_{(3 / 2) 2^{j}}} d F^{\prime}\left(\lambda_{1}, \lambda_{2}, \ldots, \lambda_{d}\right)\right)^{1 / 2}<\infty .
$$

Moreover (2.5) and (2.6) yield

$$
\sum_{j=0}^{\infty}\left(\int_{\left(\lambda_{1}, \ldots, \lambda_{d}\right)<\square_{2} j+1^{-}-\square_{2} j} d F\left(\lambda_{1}, \ldots, \lambda_{d}\right)\right)^{1 / 2}<\infty .
$$

Finally, we note that

$$
\begin{aligned}
& \left(\int_{2^{j} \leqslant|\lambda|<2^{j+1}} \ldots \int d F^{\prime}\left(\lambda_{1}, \ldots, \lambda_{d}\right)\right)^{1 / 2} \\
& \left.\leqslant\left(\int_{\left(\lambda_{1}, \ldots, \lambda_{d}\right) \in \square_{2} j+1-\square_{2} j} \ldots \int^{\prime}\left(\lambda_{1}, \lambda_{2}, \ldots, \lambda_{d}\right)\right)^{1 / 2}+\left(\int_{\left(\lambda_{1}, \ldots, \lambda_{d}\right) \in \square_{2} j-\square_{2} j-1} \ldots \int d \lambda_{1}, \ldots, \lambda_{d}\right)\right)^{1 / 2} \\
& \ldots+\left(\int_{\left(\lambda_{1}, \lambda_{2}, \ldots, \lambda_{d}\right) \in \square_{2} j-N^{\prime}+1-\square_{2} j-N^{\prime}} \ldots F^{\prime}\left(\lambda_{1}, \lambda_{2}, \ldots, \lambda_{d}\right)\right)^{1 / 2},
\end{aligned}
$$

where $N^{\prime}$ is defined by the relation $2^{N^{\prime}-1} \leqslant \sqrt{d} \leqslant 2^{N^{\prime}}$.

Consequently

$$
\begin{aligned}
\sum_{j=0}^{\infty}\left(\int_{2^{j} \leqslant|\bar{\lambda}| \leqslant 2^{j+1}} \ldots \int d F^{\prime}\left(\lambda_{1}, \ldots, \lambda_{d}\right)\right)^{1 / 2} & \\
& \leqslant N^{\prime} \sum_{j=1}^{\infty}\left(\int_{\left.\left(\lambda_{1}, \ldots, \lambda_{d}\right) \in \square_{2^{j+1}-\square_{2} j} \ldots \int^{\prime}\left(\lambda_{1}, \ldots, \lambda_{d}\right)\right)^{1 / 2}<\infty,}\right.
\end{aligned}
$$

which proves Theorem 1.

§ 3. Proof of Theorem 2. Proof of Theorem 2 can be carried out in the same way as in Nisio [7], but for the sake of completeness, we will give it with a slight modification. At first, suppose that $\left\{s_{n}\right\}$ itself is decreasing and $\sum_{n=1}^{\infty} \sqrt{s_{n}}<\infty$
Let $c(j)=c^{2^{j}}$ and put, for $j=1,2, \ldots$ :

$$
X_{j}(\bar{t})=\int_{c(j-1) \leqslant|\bar{\lambda}| \leqslant c(j)} \ldots \int^{i(\bar{t}, \bar{\lambda})} d F(\bar{\lambda}) .
$$

Since $E\left(\left|(\bar{t}+\bar{h})-x_{j}(\bar{t})\right|^{2}\right) \sim h^{2}, X_{j}(\bar{t})$ has continuous paths. For $\bar{t}=\left(t_{1}, \ldots\right.$ $\left.\ldots, t_{d}\right)$, we can write for $i=1,2, \ldots, d$, and $j=1,2, \ldots, t_{i}=\sum_{k=j+1}^{\infty} \frac{l(i, k)}{c(k)}$, where $l(i, k)$ are integers satisfying $0 \leqslant l(i, j+1)<c(j+1)$ and $0 \leqslant l(i, k)<\frac{o(k)}{o(k-1)}(k=j+2, \ldots)$. Now, putting

$$
\bar{t}_{k}=\left(\sum_{r=j+1}^{\infty} \frac{l(1, r)}{c(r)}, \ldots, \sum_{r=j+1}^{\infty} \frac{l(d, k)}{c(r)}\right),
$$

we have

$$
X_{j}(\bar{t})=X_{j}\left(\tilde{t}_{j+1}\right)+\sum_{k=j+1}^{\infty}\left(X\left(\vec{t}_{k+1}\right)-X\left(\bar{t}_{k}\right)\right) .
$$

If we denote

$$
\begin{gathered}
\eta_{j}=\max _{0<l_{i}<c(j+1)}\left|X_{j}\left(\frac{l_{1}}{c(j+1)}, \ldots, \frac{l_{d}}{c(j+1)}\right)\right| \\
\zeta(\bar{p}, \bar{q}, k)=X_{j}\left(\frac{p_{1}}{c(k)}+\frac{q_{1}}{c(k+1)}, \ldots, \frac{p_{d}}{c(k)}+\frac{q_{d}}{c(k+1)}\right)- \\
\quad-X_{j}\left(\frac{p_{1}}{c(k)}, \ldots, \frac{p_{d}}{c(k)}\right),
\end{gathered}
$$

and

$$
\theta_{k}=\max _{\substack{0 \leqslant p_{i}<c(k) \\ 1<\alpha_{i}<\frac{c(k+1)}{c(k)}}}|S(\bar{p}, \bar{q}, k)|
$$

we have

$$
\sup _{t \in \square_{1}}\left|X_{j}(\bar{t})\right| \leqslant \eta_{j}+\sum_{k=j+1}^{\infty} \theta_{k}
$$

Now, using the estimate of Delporte (Theorem 3.5.2.A, (p. 154) in [1]), we have, if we put $\sigma_{j}^{2}=F\left(S_{c(j)}\right)-T\left(S_{c(j+1)}\right)$,

$$
E\left(\eta_{j}\right) \leqslant A\{\log c(j+1)\}^{1 / 2} \sigma_{j}
$$

and

$$
\mathbb{E}\left(\theta_{k}\right) \leqslant A\left\{\log 0\left(7_{k}+1\right)\right\}^{1 / 2} \sup _{\substack{0<p_{i}<c(k) \\ 0<q_{i}<\frac{c(k+1)}{c(k)}}} E\left(|S(\bar{p}, \bar{q}, k)|^{2}\right)^{1 / 2}
$$


where $A$ is an absolute constant.

On the other hand, we have

$$
\begin{aligned}
E\left(|S(\bar{p}, \bar{q}, k)|^{2}\right) & =2 \int_{c(j-1) \leqslant|\bar{\lambda}| \leqslant c(j)}\left(1-\cos \left(\frac{\bar{q}}{c(k+1)}, \bar{\lambda}\right)\right) d F(\bar{\lambda}) \\
& \leqslant\left(\frac{c(j)}{c(k)}\right)^{2} \sigma_{j}^{2}
\end{aligned}
$$

Therefore,

$$
\mathbb{E}\left(\theta_{k}\right) \leqslant A\{\log c(k+1)\}^{1 / 2}\left(\frac{c(j)}{c(k)}\right) \sigma_{j}
$$

and consequently

$$
E(\|X\|) \leqslant \sum_{j=1}^{\infty} E\left(\left\|X_{j}\right\|\right)+E\left(\sup _{t \in \square_{1}} \mid \int_{0 \leqslant|\lambda| \leqslant 2^{a}} e^{i(\bar{t}, \bar{\lambda})} d F(\bar{\lambda}) \|\right) .
$$

The second term of the right-hand side is finite. On the other hand,

$$
\begin{aligned}
\sum_{j=1}^{\infty} E\left(\left\|X_{j}\right\|\right) & \leqslant A \sum_{j=1}^{\infty}\{\log c(j+1)\}^{1 / 2} \sigma_{j}+A \sum_{j=1}^{\infty} \sum_{k=j+1}^{\infty}\{\log c(k+1)\}^{1 / 2}\left(\frac{c(j)}{c(k)}\right) \sigma_{j} \\
& \leqslant 2 A \sum_{j=1}^{\infty} 2^{j / 2} \sigma_{j} .
\end{aligned}
$$

Since we can show that

$$
2^{j / 2} \sigma_{j} \leqslant 4 \sum_{k=2^{j-2}}^{2^{j-1}} \sqrt{s_{k}}
$$

we have

$$
E(\|X\|)<\infty,
$$

which implies the continuity of paths by Yu. K. Belyaer's theorem (soe, for example, Theorem 5 in Jain and Kallianpur [3]).

Let us now pass to the general case. Put

$$
G(A)=F(A)+\sum_{n=0}^{\infty}\left(M_{n}-s_{n}\right) \delta_{\partial S_{2} n+1}(A), \quad A \in B\left(\boldsymbol{R}^{n}\right),
$$

where $\delta_{\partial S_{2 n+1}}(\cdot)$ is the uniform probability measure concentrated on $\partial S_{2} n+1=\left\{\lambda ; \quad|\lambda|=2^{n+1}\right\}$ and $B\left(\boldsymbol{R}^{n}\right)$ are Borel sets in $\boldsymbol{R}^{n}$. We can con- struct independent stationary Gaussian processes $X_{1}$ and $X_{2}$ such that

$$
\begin{aligned}
& \varrho_{1}(\bar{t})=E\left(X_{1}(\bar{t}+\bar{s}) X_{1}(\bar{s})\right)=\int_{\boldsymbol{n}^{n}} e^{i(\bar{t}, \bar{\lambda})} d F(\bar{\lambda}), \\
& \varrho_{2}(\bar{t})=\boxplus\left(X_{2}(\bar{t}+\bar{s}) X_{2}(\bar{s})\right)=\int_{\boldsymbol{n}^{n}} e^{i(\bar{t}, \bar{\lambda})} d H(\bar{\lambda}),
\end{aligned}
$$

where $H(A)=\sum_{n=0}^{\infty}\left(M_{n}-s_{n}\right) \delta_{\theta s_{2} n+1}(A)$. Then, $G$ is the spectral measure of the covariance function of the process $X_{1}+X_{2}$ and $G\left(S_{2^{n+1}}\right)-G\left(S_{2^{n}}\right)$ $=M_{n}$. We have therefore

$$
P\left(\left\|X_{1}+X_{2}\right\|<\infty\right)=1 .
$$

Using again Delporte's lemma, we obtain

$$
P\left(\left\|X_{1}\right\|<\infty\right)=1
$$

which implies the path continuity of the process $X_{1}$.

\section{\$ 4. Examples.}

Exampus 1. Let $X$ be a real, separable, stationary Gaussian process with the covariance function $\varrho(\bar{h})=\varrho_{1}\left(h_{1}\right) \varrho_{2}\left(h_{2}\right) \ldots \varrho_{d}\left(h_{d}\right)$, where $\bar{h}$ $=\left(h_{1}, h_{2}, \ldots, h_{d}\right)$ and $\varrho_{i}$ is the covariance function of a stationary Gaussian process with a 1-dimensional parameter space. When some $\varrho_{i}$ are covariance functions which statisfy the condition of Theorem $3, X$ has discontinuous paths in virtue of Theorem 3.

Let $X=\{X(p), p \in H\}$ be a stationary Gaussian process with zero mean, where $H$ is a Hilbert space. If $\varrho(p)=E(X(p+q) X(q)), p, q \in H$, is continuous with respect to the $S$-topology (see, for example, Parthasarathy [8]), $\varrho(p)$ take the form.

$$
\varrho(p)=\int_{I I} e^{i(\lambda, p)} d W^{\prime}(\lambda)
$$

Then, wo can propose similar problems to Theorems 1 and 2.

\section{References}

[1] J. Delporto, Funclions aléatoires presque sûrement continues sur un intervalle fermé, Ann. Inst. IIonri Poincaró (B), 1 (1964), pp. 111-215.

[2] X. Fornique, Resgularite de processus ganssiens, Invontiones Math. 12 (1971) pp. $304-320$.

[3] N. C. J ain and G. Kllianpur, Oscillation function of a multiparameter Gaussian process, Nagoya Mitith. J. 47 (1972), pp. 15-28.

[4] J. P. Kahano, Some random series of funotions, 1968, Massachusetts. 
[5] M. B. Marcus, A comparison of contimuity conditions for Gaussian processes (manuscript).

[6] M. B. Marcus and L. A. Shepp, Oontinuity of Gaussian processes, Trans. Amer. Math. Soc. 151 (1970), pp. 377-391.

[7] M. Nisio, On the continuity of stationary Gaussian processes, Nagoya Math. J. 34 (1969), pp. 89-104.

[8] K. R. Parthasarathy, Probability measures on metrio spaoes, New York 1967.

[9] V. N. Sudakov, On a criterion of continuity of Gaussian sample functions, Second Japan-USSR Symposium on Prob. Th. (1972), Kyoto.

\section{Sections induced from weakly sequentially complete spaces*}

by

CHARLES F. DUNKL and DONALD E. RAMIREZ

(Charlottesville, Va.)

Ahstract. It is shown that function algebras are never weakly sequentially complete (unless finite dimensional) and then sections induced from maps from wealkly sequentially complete spaces onto function algebras are studied. As a result, it is shown that for an infinite Helson set $E$ the restriction map $\varrho$ of the Fourier algebra $A(G)$ (that is, $L^{2}(G) * L^{2}(G)$ ) of a locally compact (not necessarily abelian) group onto the space $O(E)$ of continuous functions on $E$ never admits a section $\pi$, (that is, a contin set provided $A(G) \mid X=O(E)$. A similar application to Sidon sets in the dual of set provided $A(G) \mid X=O(E)$.
a compact group is also given.

THEORwm 1. Let $A$ be a weakly sequentially complete commutative Banach algebra. If $A$ is isomorphic to a closed subalgebra $\tilde{A}$ of $O_{0}(S)$, the continuous complex-valued functions vanishing at infinity on a locally compact Hausdorff space, then $A$ is finite-dimensional.

Proof. If $\mathrm{A}$ is infinite-dimensional, then there exists an infinitedimensional separable subalgebra which is weakly sequentially complete. Thus we may assume that $A$ is separable.

If $\tilde{A}$ does not separate the points of $S$, we embed $A$ instead into $O_{0}(S / \sim)$, where for $s, t \in S, s \sim t$ if and only if $\tilde{f}(s)=\tilde{f}(t)$ for all $f \in A$. Thus we may assume that $\tilde{A}$ separates the points in. $S$ and hence in the Shilor boundary $\partial A$ (since $\partial A \subset S)$. Thus $\partial A \subset S$ is a metrizable locally compact space.

Let $P \subset \partial A \subset S$ denoto the set of peak points of $A$. The set $P$ is dense in $\partial A$. (Bishop's theorem ([6], p. 56 ) since $A$ is metrizable. It will thus suffice to show that $P$ is finite: for then $\partial A$ will be finite (and equal to $P$, and $A$ is isomorphic to $\tilde{A} \mid \partial A$.

By the Lebesgue dominated convergence theorem, given a sequence $\left\{f_{n}\right\} \subset A$ with $\left\|\tilde{f}_{n}\right\|_{\infty} \leqslant 1$ and $\tilde{f}_{n} \stackrel{n}{\rightarrow} \chi_{p}$ (the characteristic function of the set $\{p\}, p \in P$ ) pointwise on $S$, it follows that $\left\{\tilde{f}_{n}\right\}$ is weakly Oauchy in $\widetilde{A}(\cong A)$. Hence, by the weak sequential completeness of $A, \chi_{p} \in \bar{A}$. Thus $P$ consists of isolatied points.

* This research was supported in part by NSF contract GP-31483X. 